\title{
Comparaison morphométrique et enzymatique des populations de Mythimna unipuncta (Haworth) (Lep: Noctuidae) originaires de 3 îles des Açores
}

\author{
V Vieira 1 , B Pintureau 2 \\ 1 Universidade dos Açores, Departamento de Biologia, Apartado 1422, 9500 Ponta Delgada (Açores), Portugal; \\ 2 INSA-INRA, laboratoire associé Biologie 406, 20, av A-Einstein, F69621 Villeurbanne Cedex, France
}

(Reçu le 21 décembre 1992; accepté le 6 mai 1993)

\begin{abstract}
Résumé - Une étude morphométrique et une analyse électrophorétique ont été menées chez 3 populations de $M y$ thimna unipuncta originaires de l'archipel des Açores (îles de São Miguel, Santa Maria et São Jorge). Dix-neuf et 14 caractères morphométriques, portant sur les ailes, les antennes, les pattes et les genitalia mâles, ont été mesurés respectivement chez les mâles et les femelles. Après l'étude des corrélations entre ces caractères, une analyse factorielle discriminante a été menée pour séparer les 3 populations étudiées. La discrimination des populations est meilleure avec les caractères des mâles qu'avec ceux des femelles, très probablement à cause du plus faible nombre de ces derniers. Les individus vivant sur les 3 îles sont morphologiquement proches, malgré une petite divergence de ceux de São Miguel. L'étude électrophorétique, menée sur les œufs et l'hémolymphe des larves, a concerné les estérases et tétrazolium-oxydases. Une interprétation génétique a pu être effectuée au niveau de 4 loci où nous avons reconnu 15 allèles. D'après l'analyse factorielle des correspondances, menée à partir des fréquences alléliques, les 3 populations étudiées sont très proches. L'ensemble de ces résultats indique que des migrations se produisent entre les 3 îles, ou que des migrations se produisent à partir des continents européen et/ou américain, ou que les populations des diverses îles sont isolées depuis trop peu de temps pour avoir divergé de façon notable.
\end{abstract}

Mythimna unipuncta / morphométrie / électrophorèse / analyse multivariée / Açores

Summary - Morphometric and enzymatic comparison between populations of Mythimna unipuncta (Haworth) (Lep: Noctuidae) from 3 islands in the Azores. A morphometric study and an electrophoretic analysis of enzymes were carried out on 3 populations of Mythimna unipuncta from 3 islands (São Miguel, Santa Maria and São Jorge) in the Azores archipelago. Nineteen and 14 morphometric characters associated with the wings, antennae, legs and male genitalia were measured in males and females respectively. After a study of correlations between characters, a factorial discriminant analysis was carried out. Population discrimination was better for the male than for the female characters, probably due to the lower number of characters measured in the latter. We concluded that the individuals living in the 3 islands were morphologically similar, despite a small deviation in those from São Miguel. The electrophoretic study, carried out on larvae eggs and hemolymph, concerned esterase and tetrazolium-oxidase. A genetic interpretation was possible for 4 loci, where 15 alleles were identified. According to a factorial correspondence analysis carried out from allele frequencies, the 3 studied populations were found to be very close. From the results, it can be concluded that the populations are not significantly different. However, the reasons for this are not clear, and we are unable to differentiate between the 3 following hypotheses: 1) migrations occur between the 3 islands; 2) migrations occur from the European and/or American continents; or 3) populations from different islands are isolated after an insufficient period of time to have notably diverged.

Mythimna unipuncta / morphometry / electrophoresis / multivariate analysis / Azores 


\section{INTRODUCTION}

Mythimna unipuncta (Haworth) (Lepidoptera, Noctuidae) est le plus important ravageur des pâturages aux Açores. II cause des dégats estimés annuellement à $8 \%$ de la production végétale, soit environ 44 millions de francs français (Tavares, 1989). Cela explique que cette espèce soit très étudiée aux Açores, notamment sur le plan appliqué pour mettre au point une lutte intégrée.

L'objectif de cette étude est de comprendre si $M$ unipuncta, considéré comme cosmopolite (Breeland, 1958 ; Fields and McNeil, 1984 ; Bues et al, 1987), migrant (Anglade, 1969 ; Causse et Poitout, 1984; Fields et McNeil, 1984 ; Bues et al, 1987 ; McNeil, 1987) et polyphage (Metcalf et al, 1962 ; Mukerji et Guppy, 1970; Hemmerlé et al, 1981 ; Bues et al, 1987), a subi des phénomènes de différentiation dans l'archipel des Açores. Pour cela, 3 populations originaires des îles de São Miguel, Santa Maria et São Jorge (fig 1) ont été comparées à l'aide de caractères morphométriques et enzymatiques.

L'analyse des caractères morphologiques, bien que parfois peu aisée, permet d'apprécier le degré de variabilité des populations (Guillaumin, 1971 ; Cornuet et al, 1975 ; Pintureau, 1980a, b). Plusieurs descriptions morphologiques de $M$ unipuncta ont été effectuées, concernant par exemple les genitalia (Pierce, 1909 ; Franclemont, 1951 ; Breeland, 1958 ; Berio, 1985) et l'appareil reproducteur mâle et femelle (Callahan et Chapin, 1960), mais aucune mensuration morphométrique précise n'a encore été publiée. La comparaison de nos 3 populations porte sur des mensurations prises sur les ailes, les pattes, les antennes et les genitalia mâles.

L'étude de nombreux systèmes enzymatiques a été menée par électrophorèse chez beaucoup d'insectes (Pintureau, 1987). Plusieurs Lépidoptères ont ainsi été étudiés pour différencier des populations ou sous-populations (Harrison et al, 1983 ; George, 1984 ; Hughes et Zalucki, 1984 ; Zalucki et al, 1987, etc) ou des espèces morphologiquement proches (Jelnes, 1975a, b ; Pashley, 1983). D'autres auteurs, tels que Saul et al (1978), ont même remarqué que les variations de fréquences alléliques permettent de comprendre certains phénomènes écologiques. En ce qui concerne $M$ unipuncta, aucun résultat d'analyse d'enzymes n'a cependant encore été publié.

Une étude préliminaire a été menée sur 5 systèmes enzymatiques : estérases (Est), tétrazolium-oxydases (TO), lactate-deshydrogénases (LDH), glutamate-deshydrogénases (GLDH) et beta-glycérophosphate-deshydrogénases $(\beta G P D H)$. Mais seules les estérases et les tétrazolium-oxydases ont donné des résultats inter-

Fig 1

\begin{tabular}{|c|c|c|}
\hline$\bigcup_{\text {F1ares }}^{31^{\circ 12} W}$ & $28^{\circ} 36^{\prime} \mathrm{W}$ & $\begin{array}{l}26^{\circ} 00^{\prime} \mathrm{W} \\
39^{\circ} 18^{\prime} \mathrm{N} \\
\end{array}$ \\
\hline & & $38^{\circ} 11^{\prime} \mathrm{N}$ \\
\hline & & $\begin{array}{r}\text { São Higue1 } \\
37{ }^{\circ 03} \mathrm{~N}\end{array}$ \\
\hline & & Sonts Maria \\
\hline
\end{tabular}

Fig 1. Localisation des 3 îles açoriennes d'où proviennent les individus de $M$ unipuncta étudiés. 
prétables. Nous avons donc comparé nos 3 populations de $M$ unipuncta à partir des estérases de l'œuf et de l'hémolymphe du $6^{e}$ stade larvaire, et des tétrazolium-oxydases (ou superoxydedismutases ou indophénol-oxydases) de l'hémolymphe du même stade.

Les 2 systèmes enzymatiques retenus (Est et TO) sont très actifs (en particulier les estérases) et sont connus pour fournir des caractères de différentiation des espèces proches chez plusieurs groupes d'insectes (Pintureau, 1987). De telles études passent par la compréhension du déterminisme génétique des bandes électrophorétiques révélées.

\section{MATÉRIEL ET MÉTHODES}

Les individus étudiés sont les descendants d'environ 100 papillons mâles et femelles capturés aux pièges lumineux dans chacune des îles de Santa Maria (Paúl et aéroport, juin 1990), São Miguel (Arribanas et Remédios, août 1990) et São Jorge (Velas, août 1990). Certains individus utilisés descendent aussi de captures réalisées en mai et juin 1991, respectivement à São Miguel et São Jorge (mêmes localités, environ 300 individus fondateurs).

L'élevage a été conduit selon la technique de Poitout et Bues $(1970,1974)$. Les repiquages ont été effectuées avec $900 \mathrm{~L} 1$ provenant d'environ 50 pontes de chaque population et les individus ont été isolés au $3^{e}$ stade larvaire. L'ensemble du cycle s'est déroulé dans des pièces climatisées, à $25^{\circ} \mathrm{C}$ pour les stades préimaginaux et de 20 à $25^{\circ} \mathrm{C}$ pour les adultes (photophase de $16 \mathrm{~h}$ et humidité relative de 65 à $70 \%$ ). Les individus étudiés proviennent des $2^{\mathrm{e}}, 3^{\mathrm{e}}$ et $4^{\mathrm{e}}$ générations d'élevage.

Les analyses morphométriques ont été menées sur des adultes conservés dans l'alcool à $70 \%$. Trente mâles et 30 femelles de chaque population, pris au hasard, ont été étudiés.

Quatre éléments corporels ont fait l'objet de mensurations chez les 2 sexes : 1 patte postérieure, 1 antenne, 1 aile antérieure et 1 aile postérieure. Chez les mâles, nous avons en outre mesuré les genitalia. Pour cela, les papillons ont été placés dans de l'eau distillée pendant $15 \mathrm{~min}$, puis les ailes, pattes, antennes et genitalia ont été prélevés et montés sur lame dans du liquide de Faure (composition fournie par Langeron, 1949). Les mesures ont été prises, après séchage des préparations dans une étuve à $37^{\circ} \mathrm{C}$, à l'aide d'une loupe binoculaire Wild M8 munie d'un oculaire micrométrique. Elles ont ensuite été transformées en millimètres.

Seuls les caractères faciles à mesurer ont été retenus sur chaque élément corporel. Quatorze sont com-

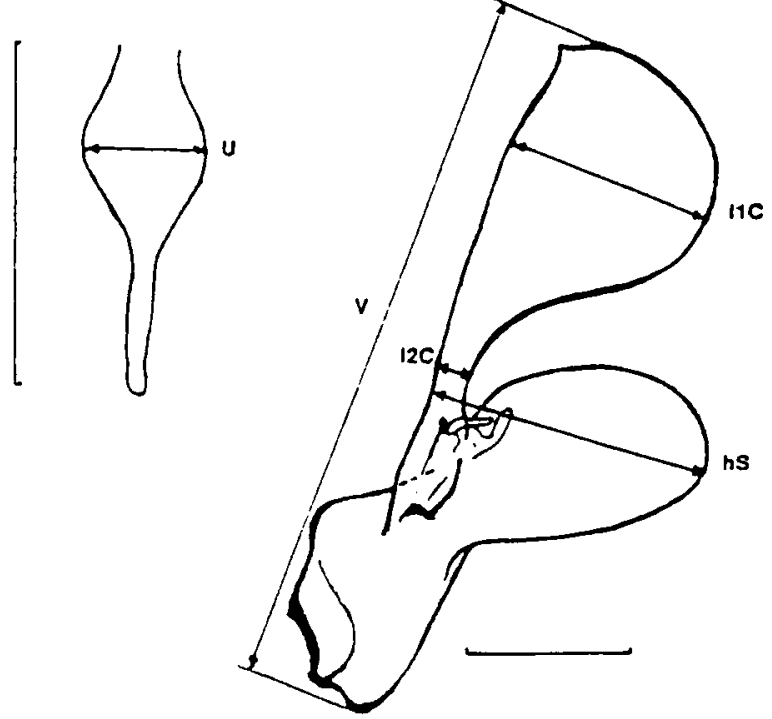

Fig 2. Caractères morphométriques mesurés sur les genitalia mâles de 3 populations de $M$ unipuncta. $U$ : largeur de l'uncus ; I1C : largeur du cucullus; 12C : largeur de la partie basale la plus étroite du cucullus; $V$ : longueur de la valva; hS : hauteur de l'extension du sacculus. L'échelle correspond à $1 \mathrm{~mm}$.

muns aux 2 sexes : longueurs de la R5, de la M3 et de la Cu1b de l'aile antérieure; longueurs de la R3, de la M3 et de la Cu1b de l'aile postérieure; Iongueurs du fémur, du tibia, du $1^{\text {er }}$ article du tarse, du plus long éperon médian du tibia et du plus long éperon terminal du tibia de la patte postérieure ; longueur du scape, largeur du scape et du pédicelle de l'antenne. Les genitalia mâles ont fait l'objet de 5 mesures supplémentaires (fig 2) : largeurs de l'uncus, du cucullus et de la partie basale la plus étroite du cucullus, longueur de la valva, hauteur de l'extension du sacculus.

Après l'étude de leurs corrélations, les caractères ont été combinés dans une analyse factorielle discriminante (AFD) de manière à séparer les trois populations étudiées.

Les analyses électrophorétiques ont été menées sur des œufs (âgés de $24 \mathrm{~h}$ au maximum) et des chenilles du $6^{e}$ stade (âgées d'environ 2 j) congelés à $-20^{\circ} \mathrm{C}$. Tous les œufs étudiés par électrophorèse proviennent de pontes différentes. Ils ont chacun été déposés dans un tube Eppendorf contenant $45 \mu \mathrm{l}$ de tampon Trudgill sucré ${ }^{\star}$ (complété par $0,0854 \%$ de phényl-thio-urée) et broyés à l'aide d'un mandrin en verre. L'hémolymphe des chenilles décongelées a été prélevée en sectionnant une fausse patte et en plaçant un tube à hématocrite sur la plaie. Environ $25 \mu \mathrm{l}$ d'hémolymhe par individu ont ainsi été récupérés puis mélangés dans un tube Eppendorf à $200 \mu$ du même tampon que précédemment.

Ces opérations se sont déroulées sur de la glace pilée. Les échantillons ont ensuite été centrifugés du-

* Pour $100 \mathrm{~cm}^{3}$ de solution aqueuse $(\mathrm{pH} 8,0), 1,314 \mathrm{~g}$ de Tris, $0,018 \mathrm{~g}$ d'acide ascorbique, $0,014 \mathrm{~g}$ de cystéine hydrochlorure et $17 \mathrm{~g}$ de saccharose. 
rant 5 minutes à $9000 \mathrm{t} / \mathrm{min}$ (température : $5^{\circ} \mathrm{C}$ ) puis stockés à $-20^{\circ} \mathrm{C}$ jusqu'au jour de l'électrophorèse. La totalité du surnageant a été utilisée dans le cas des œufs, mais seulement $35 \mu$ dans le cas de l'hémolymphe.

L'électrophorèse a été effectuée dans des plaques verticales de gel de polyacrylamide $(7 \%, \mathrm{pH} 8,9)$ selon une méthode déjà décrite (Pintureau et Babault, 1981 ; Pintureau, 1987 ; Pintureau et al, 1991) avec quelques modifications: le voltage a été de $150 \mathrm{~V}$ pendant $30 \mathrm{~min}$ puis soit de $300 \mathrm{~V}$ pendant $90 \mathrm{~min}$ (estérases des œufs), ou de $200 \mathrm{~V}$ durant $10 \mathrm{~min}$ suivis de $450 \mathrm{~V}$ pendant $90 \mathrm{~min}$ (estérases de l'hémolymphe), ou de $300 \mathrm{~V}$ durant $80 \mathrm{mn}$ (TO de l'hémolymphe); la révélation des estérases a été réalisée avec $120 \mathrm{mg}$ de 2-naphtyl-acétate et $60 \mathrm{mg}$ de 1naphtyl-acétate; la durée de révélation a été de 60 min (estérases des œufs et TO de l'hémolymphe) ou de $90 \mathrm{~min}$ (estérases de l'hémolymphe).

La révélation des estérases a été effectuée individuellement sur 105 œufs (32 de São Miguel, 35 de Santa Maria et 38 de São Jorge) et sur l'hémolymphe de 181 chenilles du dernier stade (61 de São Miguel, 48 de Santa Maria et 72 de São Jorge). Celle des tétrazolium-oxydases n'a été effectuée que sur l'hémolymphe de 107 chenilles du dernier stade (40 de São Miguel, 34 de Santa Maria et 33 de São Jorge).

Les locus ont été nommés de manière à indiquer l'enzyme codée (Est ou TO), le stade de l'insecte où l'enzyme fonctionne ( $\mathrm{W}$ pour œuf et $\mathrm{H}$ pour hémolymphe du dernier stade larvaire) et le numéro de la zone colorée repérée sur les électrophorégrammes (2 ou 3). Une analyse factorielle des correspondances (AFC) a été menée à partir des fréquences alléliques de manière à séparer les 3 populations étudiées.

\section{RÉSULTATS}

\section{Étude morphométrique}

Peu de différences entre populations apparaissent de manière évidente (tableau I), les plus nette concernant M3 A1, Cu1b A1 et M3 A2 chez les femelles.

Nous avons étudié les corrélations entre caractères chez les mâles et les femelles en rassemblant les 3 populations $(n=90)$. Les variables étudiées chez les mâles sont en majorité liées (tableau II). Les exceptions concernent la longueur du scape et 16 autres caractères, la largeur du pédicelle et 13 autres caractères, et la largeur de la partie basilaire du cucullus et 13 autres caractères. La liaison la plus forte est constatée entre Cu1b et M3 (aile antérieure) et la liaison la plus faible entre $V$ (genitalia) et LSc (antenne). Aucune corrélation négative n'est notée.
Chez les femelles, beaucoup de variables sont également liées (tableau III). Les exceptions concernent encore la longueur du scape et 4 autres caractères, et la largeur du pédicelle et 4 autres caractères. La corrélation la plus forte est constatée entre M3 et R5 (aile antérieure) et la plus faible entre Ep2 (patte) et IP (antenne). Aucune n'est négative.

La totalité de l'information traitée par les AFD est absorbée par 2 axes discriminants : $86,1 \%$ pour l'axe 1 et 13,9\% pour l'axe 2 chez les mâles ; $71,9 \%$ pour l'axe 1 et $28,1 \%$ pour l'axe 2 chez les femelles.

La discrimination des 3 populations obtenue avec les mâles (fig 3 ) est meilleure que celle obtenue avec les femelles (fig 4), très probablement à cause du plus grand nombre de caractères utilisés. Un fort chevauchement existe entre les populations de Santa Maria et de São Jorge. Les individus vivant sur les 3 îles sont donc morphologiquement proches, malgré une petite divergence de ceux de São Miguel.

Les vecteurs propres (tableau IV) nous renseignent sur la signification de chaque axe et sur l'importance relative des divers caractères utilisés. Chez les mâles, les variables les plus importants sont LSc, ISc (antenne) et I2C (genitalia) pour discriminer la population de São Miguel des 2 autres (axe 1), et LSc, ISc (antenne), U (genitalia) et Ep1 (patte) pour discriminer les populations de Santa Maria et São Jorge (axe 2). Chez

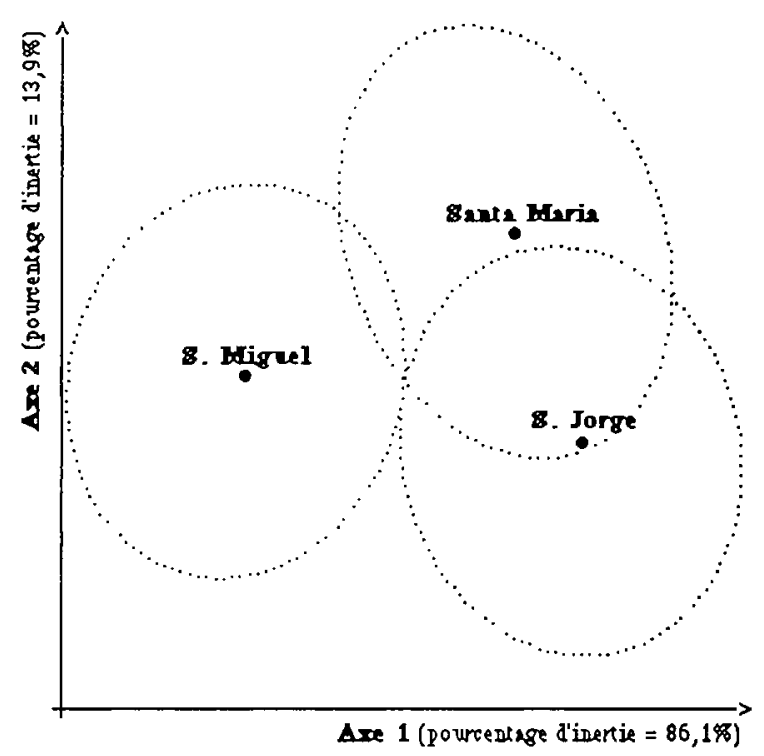

Fig 3. Analyse factorielle discriminante (AFD) effectuée à partir de 19 caractères morphométriques mesurés chez les mâles de 3 populations açoriennes de $M$ unipuncta. $N=30$ par population. Les ellipses contiennent $95 \%$ des observations. 
Tableau I. Valeurs moyennes ( \pm écarts types), en $\mathrm{mm}$, des 19 caractères morphométriques mesurés chez des mâles et des 14 caractères mesurés chez des femelles de $M$ unipuncta ( 3 populations açoriennes).

\begin{tabular}{|c|c|c|c|c|c|c|}
\hline \multirow[t]{2}{*}{ Caractères } & \multicolumn{3}{|c|}{ Mâles } & \multicolumn{3}{|c|}{ Femelles } \\
\hline & São Miguel & Sta Maria & São Jorge & São Miguel & Sta Maria & São Jorge \\
\hline \multicolumn{7}{|c|}{ Aile antérieure } \\
\hline R5 A1 & $8,54 \pm 0,63$ & $8,17 \pm 0,42$ & $8,18 \pm 0,54$ & $8,89 \pm 0,48$ & $8,04 \pm 0,73$ & $8,69 \pm 0,63$ \\
\hline M3 A1 & $6,33 \pm 0,50$ & $6,01 \pm 0,35$ & $5,99 \pm 0,41$ & $6,60 \pm 0,43$ & $5,92 \pm 0,53$ & $6,37 \pm 0,45$ \\
\hline Cu1b A1 & $7,46 \pm 0,55$ & $7,08 \pm 0,41$ & $7,02 \pm 0,51$ & $7,81 \pm 0,44$ & $6,87 \pm 0,58$ & $7,43 \pm 0,54$ \\
\hline \multicolumn{7}{|c|}{ Aile postérieure } \\
\hline R3 A2 & $6,79 \pm 0,53$ & $6,48 \pm 0,46$ & $6,48 \pm 0,45$ & $7,04 \pm 0,47$ & $6,27 \pm 0,60$ & $6,74 \pm 0,53$ \\
\hline M3 A2 & $6,03 \pm 0,51$ & $5,60 \pm 0,44$ & $5,67 \pm 0,46$ & $6,29 \pm 0,43$ & $5,40 \pm 0,50$ & $5,96 \pm 0,43$ \\
\hline Cu1b A2 & $7,03 \pm 0,56$ & $6,78 \pm 0,47$ & $6,74 \pm 0,47$ & $7,48 \pm 0,40$ & $6,50 \pm 0,52$ & $7,06 \pm 0,52$ \\
\hline \multicolumn{7}{|c|}{ Patte postérieure } \\
\hline $\mathrm{F}$ & $3,48 \pm 0,28$ & $3,38 \pm 0,21$ & $3,39 \pm 0,27$ & $3,62 \pm 0,20$ & $3,28 \pm 0,25$ & $3,46 \pm 0,26$ \\
\hline $\mathrm{Ti}$ & $4,40 \pm 0,49$ & $4,06 \pm 0,28$ & $4,04 \pm 0,41$ & $4,60 \pm 0,74$ & $3,96 \pm 0,34$ & $4,24 \pm 0,37$ \\
\hline $\mathrm{Ta}$ & $2,61 \pm 0,34$ & $2,49 \pm 0,22$ & $2,38 \pm 0,22$ & $2,56 \pm 0,30$ & $2,27 \pm 0,27$ & $2,41 \pm 0,32$ \\
\hline Ep1 & $1,81 \pm 0,09$ & $1,68 \pm 0,08$ & $1,73 \pm 0,12$ & $1,72 \pm 0,11$ & $1,57 \pm 0,13$ & $1,65 \pm 0,18$ \\
\hline Ep2 & $1,65 \pm 0,09$ & $1,57 \pm 0,08$ & $1,56 \pm 0,12$ & $1,54 \pm 0,96$ & $0,48 \pm 0,14$ & $1,50 \pm 0,14$ \\
\hline \multicolumn{7}{|l|}{ Antenne } \\
\hline LSc & $0,25 \pm 0,02$ & $0,29 \pm 0,03$ & $0,32 \pm 0,02$ & $0,27 \pm 0,02$ & $0,27 \pm 0,03$ & $0,30 \pm 0,03$ \\
\hline ISc & $0,47 \pm 0,02$ & $0,49 \pm 0,02$ & $0,48 \pm 0,02$ & $0,48 \pm 0,02$ & $0,46 \pm 0,03$ & $0,48 \pm 0,02$ \\
\hline IP & $0,25 \pm 0,02$ & $0,25 \pm 0,02$ & $0,26 \pm 0,03$ & $0,24 \pm 0,01$ & $0,24 \pm 0,02$ & $0,26 \pm 0,03$ \\
\hline \multicolumn{7}{|l|}{ Genitalia } \\
\hline V & $4,34 \pm 0,21$ & $4,28 \pm 0,16$ & $4,26 \pm 0,22$ & & & \\
\hline $\mathrm{I1C}$ & $1,22 \pm 0,11$ & $1,25 \pm 0,08$ & $1,22 \pm 0,11$ & & & \\
\hline $12 \mathrm{C}$ & $0,23 \pm 0,02$ & $0,24 \pm 0,01$ & $0,24 \pm 0,02$ & & & \\
\hline $\mathrm{hS}$ & $1,87 \pm 0,10$ & $1,85 \pm 0,11$ & $1,75 \pm 0,24$ & & & \\
\hline $\mathrm{U}$ & $0,38 \pm 0,04$ & $0,38 \pm 0,03$ & $0,37 \pm 0,04$ & & & \\
\hline
\end{tabular}

A1: aile antérieure ; A2: aile postérieure ; R5, R3, M3 et Cu1b : longueur de certaines nervures radiales, médianes et cubitales ; F: longueur du fémur ; $\mathrm{T}$ : longueur du tibia ; Ta : Iongueur du $1^{\mathrm{\theta} r}$ article du tarse ; EP1 et Ep2: longueur du plus long éperon médian et terminal du tibia ; LSc et ISc : longueur et largeur du scape ; IP: largeur du pédicelle; V, 11C, I2C, hS et U : voir fig 1.

les femelles, ces variables sont LSc, IP (antenne) et Ep2 (patte) pour discriminer les populations de São Miguel et Santa Maria (axe 1), et LSc et ISc (antenne) pour discriminer la population de $S$ Jorge des 2 autres (axe 2).

\section{Étude enzymatique}

La révélation des estérases des œufs et de l'hémolymphe des chenilles du dernier stade a montré qu'il existe 3 zones principales différant par leur pouvoir migratoire et leur coloration. Ces zones ne sont pas homologues chez les 2 stades étudiés. Les œufs fournissent 3 zones. La première (Rf aux environs de 0,27), fonctionnant surtout avec le 2-naphtyl-acétate, et la troisième
(Rf aux environs de 0,50), fonctionnant surtout avec le 1-napthyl-acétate, sont faibles et très peu lisibles. La deuxième ( $\mathrm{Rf}$ compris entre 0,35 et 0,42$)$, fonctionnant surtout avec le 2-naphtylacétate, a par contre pu être analysée (fig 5A). L'hémolymphe fournit également 3 zones. La première (Rf compris entre 0,68 et 0,72 ), fonctionnant surtout avec le 2-naphtyl-acétate, est très difficile à interpréter. À l'inverse, la deuxième (Rf compris entre 0,78 et 0,84 ), fonctionnant surtout avec le 2-naphtyl-acétate, et la troisième (Rf compris entre 0,84 et 0,96 ), fonctionnant surtout avec le 1-naphtyl-acétate, présentent un fort polymorphisme qui a pu être analysé (fig 5B, C).

La révélation des tétrazolium-oxydases de l'hémolymphe des chenilles du dernier stade 


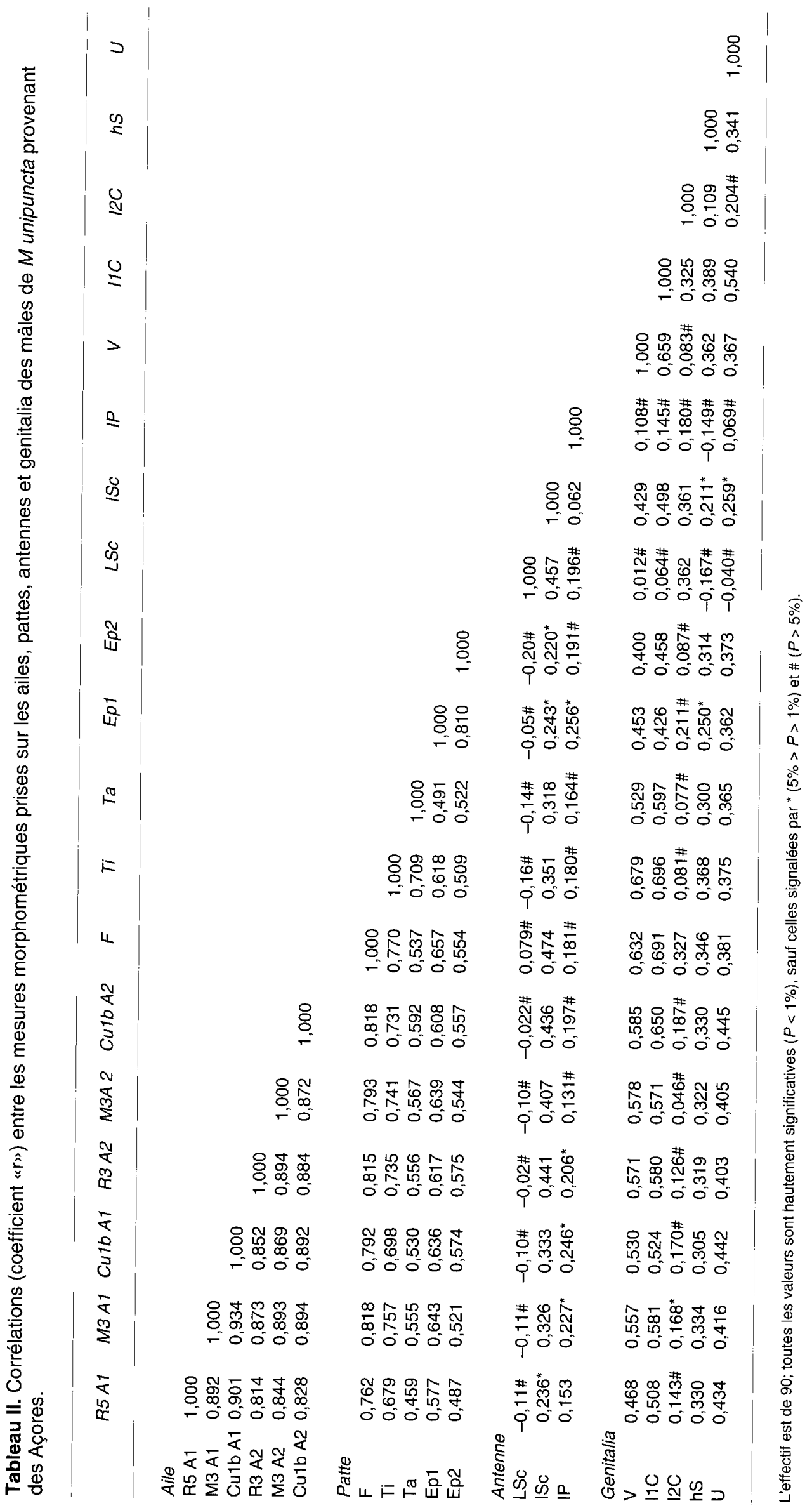




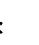

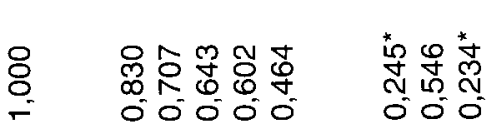

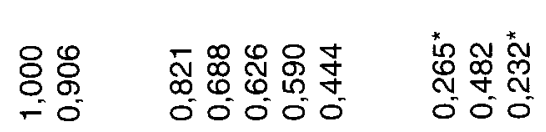

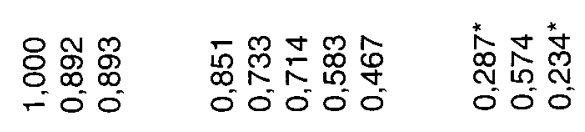

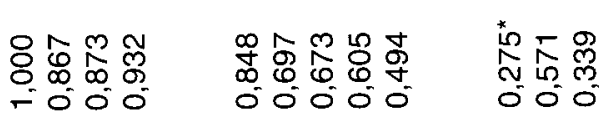

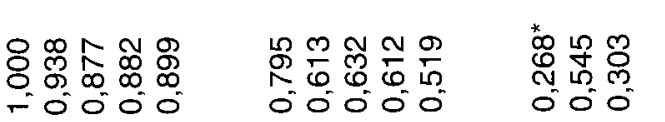

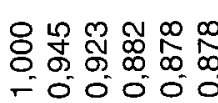

$\begin{array}{llll}0 & 0 & 0 \\ 0 & 0 & 0 \\ 0 & 0 & 0 \\ 0 & 0 & 0 \\ 0 & 0 & 0 & 0 \\ 0 & 0\end{array}$

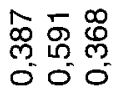

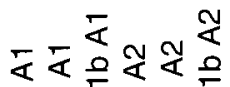

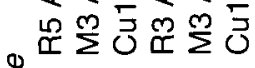




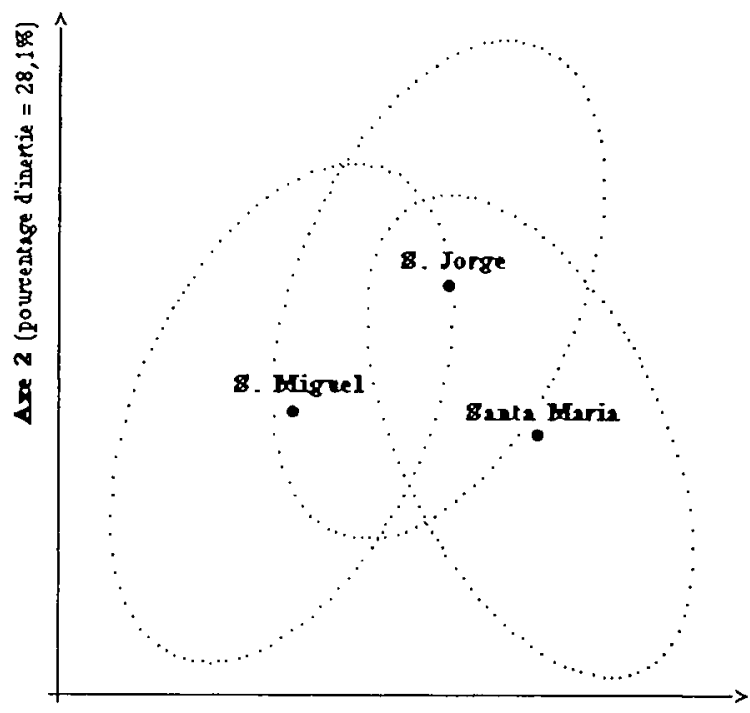

Are 1 (pourcesuge d'inerie $=71,9 \%$ )

Fig 4. Analyse factorielie discriminante (AFD) effectuée à partir de 14 caractères morphométriques mesurés chez les femelles de 3 populations açoriennes de $M$ unipuncta. $\mathrm{N}=$ 30 par population. Les ellipses contiennent $95 \%$ des observations. montre qu'il existe 2 zones différant par leur pouvoir migratoire, la première $(\mathrm{Rf}$ aux environs de 0,26 ) est faible et très difficilement analysable, tandis que la deuxième ( $\mathrm{Rf}$ compris entre 0,58 et 0,64 ) est bien visible et facile à analyser (fig 5D).

Nous avons relevé 2 allèles au niveau du locus TO2, 3 au niveau des locus Est W2 et Est $\mathrm{H} 2$ et 7 au niveau du locus Est H3. Ils ont été nommés par un chiffre : 100 pour l'allèle le plus fréquent dans la population de référence (São Miguel) et un chiffre inférieur ou supérieur à 100 pour les autres allèles, selon la mobilité relative des bandes qu'ils fournissent (tableau V). Les individus homozygotes se caractérisent par une seule bande (Est W2, Est H2, TO2) ou par 2 bandes (Est $H 3$ ), et les hétérozygotes par 2 bandes (Est W2, Est H2, TO2) ou par 4 bandes (Est H3) (fig 5A, B, C, D). Toutes les enzymes étudiées sont donc des monomères. La tre $^{\text {re }}$ bande, correspondant à un génotype homozygote sur Est $\mathrm{H} 3$, est moins intense que la $2^{\mathrm{e}}$ et provient certainement d'un produit de dégradation.

Tableau IV. Vecteurs propres correspondant aux axes 1 et 2 des AFD effectuées sur 3 populations açoriennes de $M$ unipuncta à partir de 19 caractères morphométriques des mâles ou de 14 caractères des femelles.

\begin{tabular}{|c|c|c|c|c|c|c|c|}
\hline \multicolumn{4}{|c|}{ Mâles } & \multicolumn{4}{|c|}{ Femelles } \\
\hline \multicolumn{2}{|c|}{ Axe 1} & \multicolumn{2}{|c|}{ Axe 2} & \multicolumn{2}{|c|}{ Axe 1} & \multicolumn{2}{|c|}{ Axe 2} \\
\hline Variables & Vecteurs & Variables & Vecteurs & Variables & Vecteurs & Variables & Vecteurs \\
\hline LSc & 0,880 & $1 \mathrm{Sc}$ & 0,760 & LSc & 0,770 & $\mathrm{LSc}$ & 0,899 \\
\hline $12 \mathrm{C}$ & 0,296 & $\mathrm{U}$ & 0,284 & $\mathrm{IP}$ & 0,503 & ISc & 0,398 \\
\hline ISc & 0,251 & Ep2 & 0,110 & Ep2 & 0,200 & IP & 0,125 \\
\hline $11 C$ & 0,159 & $12 \mathrm{C}$ & 0,049 & M3 A1 & 0,122 & M3 A2 & 0,037 \\
\hline IP & 0,123 & $\mathrm{Ta}$ & 0,041 & R3 A2 & 0,060 & $\mathrm{R} 5 \mathrm{~A} 1$ & 0,015 \\
\hline Ep2 & 0,049 & M3 A1 & 0,040 & R5 A1 & 0,032 & $\mathrm{Ta}$ & 0,015 \\
\hline U & 0,042 & hS & 0,038 & $\mathrm{Ti}$ & 0,010 & Ep1 & 0,006 \\
\hline Cu1b A2 & 0,003 & $\mathrm{~F}$ & 0,032 & $\mathrm{Ta}$ & 0,008 & Cu1b A2 & 0,004 \\
\hline M3 A1 & 0,029 & Cu1b A1 & 0,016 & $\mathrm{~F}$ & $-0,030$ & M3 A1 & 0,004 \\
\hline R5 A1 & 0,027 & $11 \mathrm{C}$ & 0,009 & Cu1b A2 & $-0,091$ & $\mathrm{Ti}$ & 0,001 \\
\hline M3 A2 & $-0,001$ & $\mathrm{R} 3 \mathrm{~A} 2$ & 0,008 & Cu1b A1 & $-0,120$ & Cu1b A1 & $-0,015$ \\
\hline V & $-0,004$ & Cu1b A2 & 0,000 & M3 A2 & $-0,121$ & $\mathrm{R} 3 \mathrm{~A} 2$ & $-0,026$ \\
\hline $\mathrm{F}$ & $-0,009$ & V & $-0,005$ & Ep1 & $-0,153$ & Ep2 & $-0,078$ \\
\hline $\mathrm{R} 3 \mathrm{~A} 2$ & $-0,013$ & R5 A1 & $-0,006$ & ISc & $-0,177$ & $\mathrm{~F}$ & $-0,080$ \\
\hline $\mathrm{Ta}$ & $-0,019$ & $\mathrm{Ti}$ & $-0,028$ & & & & \\
\hline $\mathrm{Ti}$ & $-0,024$ & M3 A2 & $-0,067$ & & & & \\
\hline hS & $-0,033$ & IP & $-0,096$ & & & & \\
\hline Cu1b A1 & $-0,051$ & Ep1 & $-0,296$ & & & & \\
\hline Ep1 & $-0,153$ & LSc & $-0,467$ & & & & \\
\hline
\end{tabular}



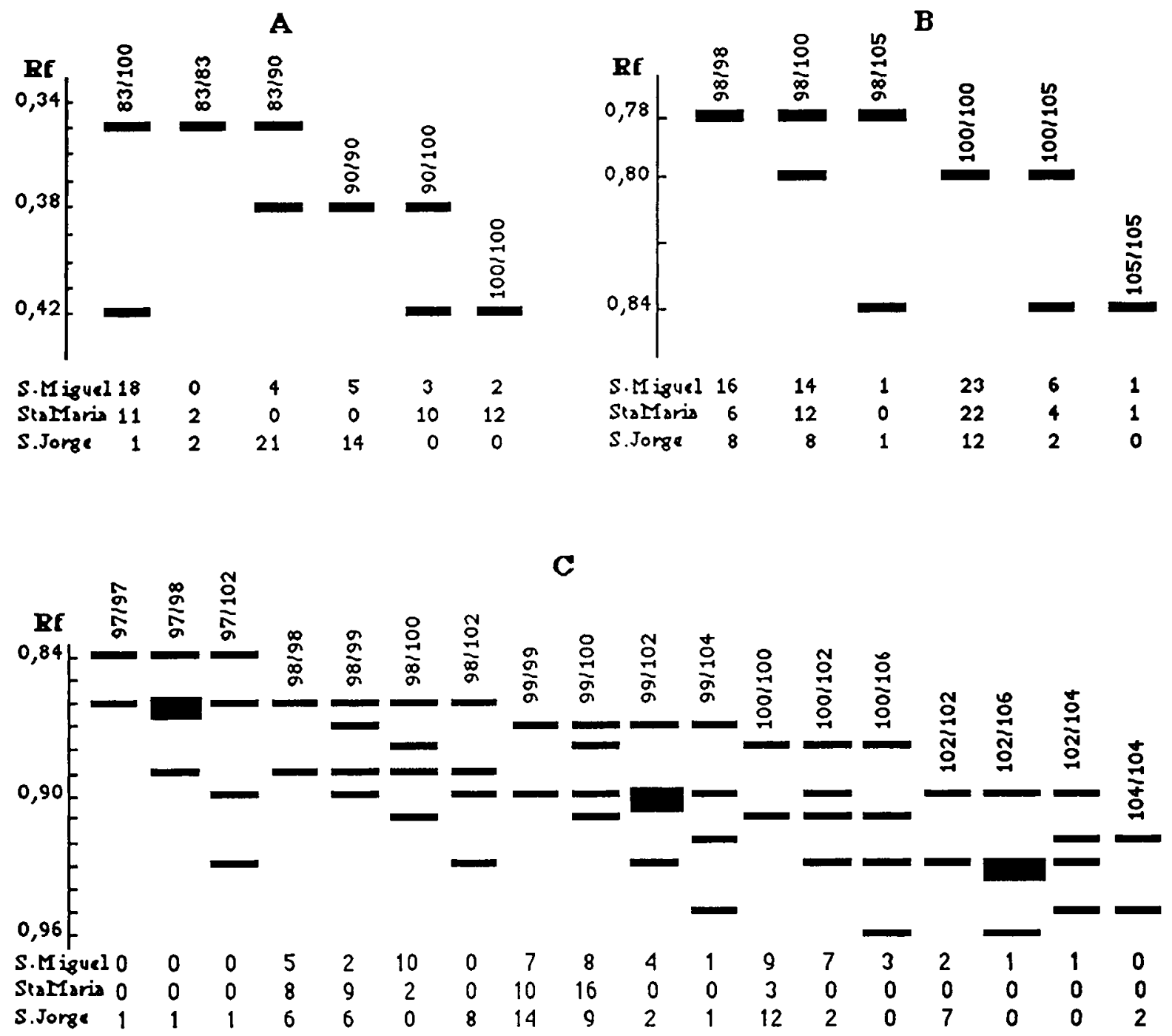

D

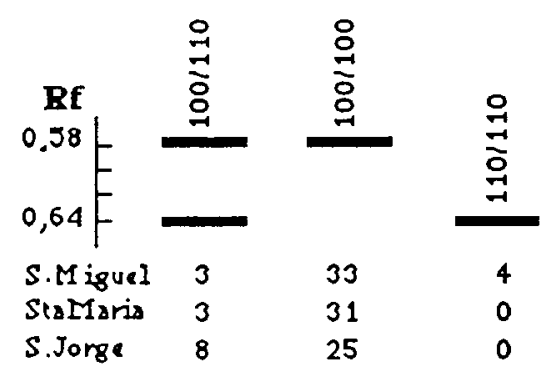

Fig 5. Phénotypes observés au niveau des locus EST W2 (A), EST H2 (B), EST H3 (C) et TO2 (D) chez 3 populations de $M$ unipuncta. Les nombres situés au-dessus des bandes indiquent les génotypes, ceux situés au-dessous indiquent les effectifs.

Les fréquences alléliques figurent dans le tableau $V$. Nous constatons qu'il existe des différences entre les populations, notamment au niveau de Est W290 (fréquence élevée à São Jorge), Est W2100 (fréquence élevée à Santa Maria) et Est H3102 (absent à Santa Maria).
Nous avons testé l'écart à la panmixie ou la déviation par rapport à l'équilibre de HardyWeinberg au niveau des 3 loci codant pour des estérases, à l'aide du test $\chi^{2}$ (comparaison des effectifs phénotypiques observés et théoriques). En ce qui concerne les locus Est W2 et Est H2, 
Tableau V. Fréquences alléliques aux 4 loci étudiés chez 3 populations de $M$ unipuncta.

\begin{tabular}{|c|c|c|c|c|}
\hline Locus & Allèles & São Miguel & Sta Maria & São Jorge \\
\hline EST W2 & $\begin{array}{r}83 \\
90 \\
100\end{array}$ & $\begin{array}{l}0,344( \pm 0,116) \\
0,266( \pm 0,108) \\
0,390( \pm 0,119) \\
(n=32)\end{array}$ & $\begin{array}{l}0,214( \pm 0,096) \\
0,143( \pm 0,082) \\
0,643( \pm 0,112) \\
(n=35)\end{array}$ & $\begin{array}{l}0,342( \pm 0,107) \\
0,645( \pm 0,108) \\
0,013( \pm 0,025) \\
(n=38)\end{array}$ \\
\hline EST H2 & $\begin{array}{r}98 \\
100 \\
105\end{array}$ & $\begin{array}{l}0,385( \pm 0,086) \\
0,541( \pm 0,088) \\
0,074( \pm 0,046) \\
(n=61)\end{array}$ & $\begin{array}{l}0,266( \pm 0,091) \\
0,667( \pm 0,097) \\
0,067( \pm 0,052) \\
(n=45)\end{array}$ & $\begin{array}{l}0,403( \pm 0,122) \\
0,548( \pm 0,124) \\
0,049( \pm 0,054) \\
(n=31)\end{array}$ \\
\hline EST H3 & $\begin{array}{r}97 \\
98 \\
99 \\
100 \\
102 \\
104 \\
106\end{array}$ & $\begin{array}{l}0 \\
0,183( \pm 0,069) \\
0,242( \pm 0,077) \\
0,383( \pm 0,087) \\
0,142( \pm 0,062) \\
0,017( \pm 0,023) \\
0,033( \pm 0,032) \\
(n=60)\end{array}$ & $\begin{array}{l}0 \\
0,469( \pm 0,100) \\
0,469( \pm 0,100) \\
0,250( \pm 0,087) \\
0 \\
0 \\
0 \\
(n=48)\end{array}$ & $\begin{array}{l}0,028( \pm 0,027) \\
0,188( \pm 0,064) \\
0,319( \pm 0,076) \\
0,243( \pm 0,070) \\
0,187( \pm 0,064) \\
0,035( \pm 0,030) \\
0 \\
(n=72)\end{array}$ \\
\hline TO2 & $\begin{array}{l}100 \\
110\end{array}$ & $\begin{array}{l}0,862( \pm 0,076) \\
0,138( \pm 0,076) \\
(n=40)\end{array}$ & $\begin{array}{l}0,956( \pm 0,049) \\
0,044( \pm 0,049) \\
(n=34)\end{array}$ & $\begin{array}{l}0,879( \pm 0,079) \\
0,121( \pm 0,079) \\
(n=33)\end{array}$ \\
\hline
\end{tabular}

$n$ : Nombre d'individus analysés. Les fréquences sont suivies de leur intervalle de confiance au seuil de $95 \%$ $(1,96 \sqrt{\text { fréquence (1-fréquence) } / 2 n}$.

les populations de Santa Maria et São Jorge sont en équilibre, tandis que la population de São Miguel ne l'est pas. Au niveau du locus Est H3, toutes les populations sont en revanche déséquilibrées (tableau VI). De tels déséquilibres peuvent cependant provenir de problèmes d'échantillonnage plutôt que d'un réel écart par rapport à la panmixie.
Le $1^{\mathrm{er}}$ axe de I'AFC absorbe $82,7 \%$ de l'inertie totale et le $2^{e} 17,3 \%$. Cette analyse montre que les 3 populations sont proches, les plus semblables étant celles de São Miguel et Santa Maria, et les moins semblables celles de Santa Maria et São Jorge (fig 6). En négligeant les allèles rares, la population de São Miguel est surtout caractérisée par la présence des allèles Est

Tableau VI. Valeurs des $\chi^{2}$ testant l'équilibre de Hardy-Weinberg au niveau de 3 loci chez 3 populations de $M$ unipuncta.

\begin{tabular}{|c|c|c|c|c|c|c|c|c|c|}
\hline \multirow[b]{2}{*}{ Locus } & \multicolumn{3}{|c|}{ São Miguel } & \multicolumn{3}{|c|}{ Santa Maria } & \multicolumn{3}{|c|}{ São Jorge } \\
\hline & $d d l$ & $\chi^{2}$ & Déséquilibre & $d d l$ & $\chi^{2}$ & Déséquilibre & $d d l$ & $\chi^{2}$ & Déséquilibre \\
\hline Est W2 & 3 & $21,327^{\star \star}$ & Excès de $83 / 100$ & 2 & 3,950 & & 1 & 2,362 & \\
\hline Est H2 & 3 & $13,066^{\star \star}$ & Déficit de $98 / 100$ & 2 & 1,999 & & 2 & 4,887 & \\
\hline Est H3 & 8 & $16,369^{\star}$ & $\begin{array}{l}\text { Excès de 99/99 } \\
\text { Déficit de 98/99 }\end{array}$ & 3 & $9,027^{\star}$ & $\begin{array}{l}\text { Excès de 99/100 } \\
\text { Déficit de } 98 / 100\end{array}$ & 8 & $46,922^{\star \star}$ & $\begin{array}{l}\text { Excès de } 100 / 100 \\
\text { et de } 99 / 99\end{array}$ \\
\hline
\end{tabular}

Certains effectifs génotypiques (théoriques et observés) ont été regroupés avec d'autres lorsqu'ils n'atteignaient que 1 ou parfois 2. * : $0,05>P>0,01 ;{ }^{\star \star}: P<0,01$; ddl : degrés de liberté. 


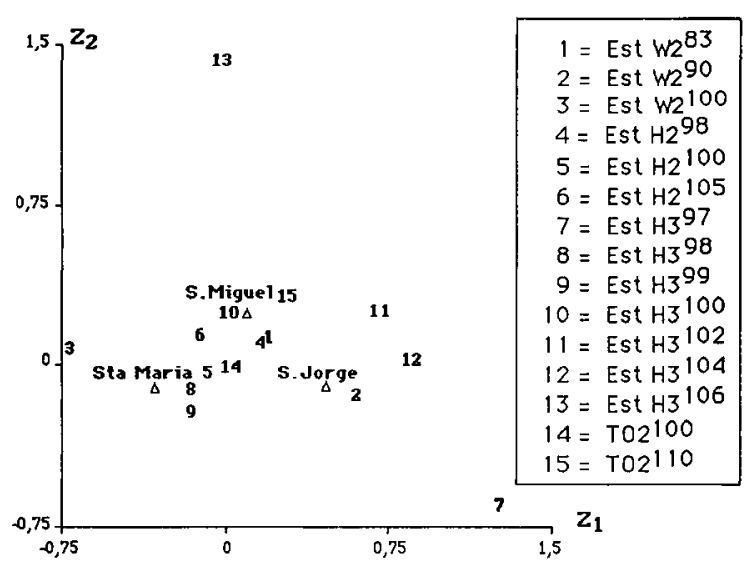

Fig 6. Analyse factorielle des correspondances entre les fréquences de 15 allèles (locus EST W2, EST H2, EST H3 et TO2) et 3 populations de $M$ unipuncta (São Miguel, Sta Maria et São Jorge).

H3100 et TO2110, celle de Santa Maria par la présence des allèles Est $\mathrm{H} 398$ et Est H399, et celle de São Jorge par la présence de l'allèle Est W290.

\section{DISCUSSION}

Les populations de $M$ unipuncta présentes sur 3 îles de l'archipel des Açores sont morphologiquement et biochimiquement très semblables. Nous pouvons alors supposer qu'un certain flux génique, jouant un rôle homogénéisant des populations, existe entre les îles étudiées. II est dans ce cas probable que ce flux soit plus important entre les îles proches (Santa Maria et São Miguel, par exemple) qu'entre celles éloignées par plusieurs centaines de kilomètres (Santa Maria et São Jorge, par exemple). Deux autres hypothèses ne sont toutefois pas à rejeter. II se pourrait qu'aucun flux n'existe entre les îles açoriennes, mais que des individus arrivent soit du continent européen, soit du continent américain. II se pourrait aussi qu'aucun flux n'existe mais que les populations de $M$ unipuncta des divers îles soient isolées depuis trop peu de temps pour avoir sensiblement divergé.

L'analyse de ces éventuels flux, qui ne correspondent pas nécessairement à des phénomènes migratoires importants, demandera à être approfondie. Pour cela, il faudra élargir l'échantillonnage à toutes les îles açoriennes et aux régions continentales les plus proches. Au niveau morphologique, nous savons déjà que les mâles des Açores ont des genitalia légèrement plus petits que ceux des individus du continent Nordaméricain (Franclemont, 1951). Des études en cours de McNeil et Vieira, concernant non seulement les genitalia mais aussi la surface des ailes d'une population du Canada et de la population de l'île de Flores, confirment d'ailleurs de telles différences. Au niveau génétique, d'autres loci devront être analysés. Deux systèmes enzymatiques et 4 loci sont en effet insuffisants pour représenter l'ensemble du génome. Nous pourrons aussi avoir recours à une technique plus sensible que l'électrophorèse des enzymes, qui est loin de détecter toutes les mutations (Saul et al, 1978 ; Crouau-Roy, 1986). L'étude du polymorphisme de l'ADN mitochondrial à l'aide d'enzymes de restriction serait par exemple très utile.

Ces études constituent un préalable indispensable à la réalisation d'un modèle prédictif des pullulations de $M$ unipuncta dans l'archipel des Açores, modèle destiné à combattre plus aisément ce ravageur des graminées. La faible divergence des individus des 3 îles étudiées, qu'une analyse des caractères biologiques (en préparation) tend à confirmer, indique déjà qu'un modèle unique est envisageable. Celui-ci suppose toutefois une meilleure compréhension du fonctionnement des populations et demande encore beaucoup de recherches.

\section{RÉFÉRENCES}

Anglade $P$ (1969) Première observations de déplacements orientés de Noctuelles et de Sphingides dans une haute vallée pyrénéenne par recapture d'insectes marqués. Bull Soc Entomol Fr 74, 59-63

Berio E (1985) Lepidoptera noctuidae. I. Generalita, Hadeninae, Cucullinae. In: Fauna d'Italia, Calderini, Bologna, 363-365

Breeland SG (1958) Biological studies on the armyworm, Pseudaletia unipuncta (Haworth), in Tennessee (Lepidoptera: Noctuidae). J Tenn Acad Sci 33, 263-347

Bues R, Poitout S, Robin JC, Anglade P (1987) Études en conditions contrôlées des limites thermiques au développement de Mythimma unipuncta Haw (Lep Noctuidae). Acta Oecol 8, 79-89

Callahan PS, Chapin JB (1960) Morphology of the reproductive systems and mating in two representative members of the family Noctuidae, Pseudaletia unipuncta and Peridroma saucia, with comparison to Heliothis zea. Ann Entomol Soc Am 53, 763-782

Causse R, Poitout $S$ (1984) Mise en évidence et étude de l'orientation des vols migratoires de certains Lépidoptères Noctuidae. In: La Vision chez les Invertébrés. Actes du Colloque International 
CNRS, Lyon, 21-23 sept 1983 (R Clement, R Ramousse, eds), CNRS, Paris, 198-202

Cornuet JM, Fresnaye J, Tassencourt L (1975) Discrimination et classification de populations d'abeilles à partir de caractères biométriques. Apidologie 6, 145-187

Crouau-Roy B (1986) Structure génétique des populations et des espèces chez des Coléoptères troglobies (Speonomus), contribution à l'étude des mécanismes de la spéciation. Thèse doctorat d'État, université des sciences et techniques du Languedoc, $250 \mathrm{p}$

Fields PC, McNeil JN (1984) The overwintering potential of true armyworm Pseudaletia unipuncta (Lep Noctuidae) population in Quebec. Can Ent 116, 1647-1652

Franclemont JG (1951) The species of the Leucania unipuncta group, with a discussion of the genetic names for the various segregates of Leucania in North America. Proc Ent Soc Wash 53, 57-85

George C (1984) Allozyme variation in natural populations of Lymantria dispar (Lepidoptera). Genet Sel Evol 16, 1-14

Guillaumin M (1971) Étude de la variabilité morphologique et biométrique des populations naturelles de Pyrgus malvae $\mathrm{L}$ et $P$ malvoides Elv et Edw dans leur zone de contact (Lep Hesperiidae). Discussion sur les rapports taxonomiques entre ces 2 formes. Vie Milieu (Ser C) 22, 91-151

Harrison RG, Wintermeyer SF, Odell TM (1983) Patterns of genetic variation within and among gypsy moth, Lymantria dispar (Lepidoptera: Lymantriidae), populations. Ann Entomol Soc Am 76, 652-656

Hemmerlé A, Chaffurin C, Rocque B (1981) Un ravageur des maïs et des prairies du Sud-Ouest, la noctuelle des Graminées. Phytoma 329, 25-28

Hughes JM, Zalucki MP (1984) Genetic variation in a continuously breeding population of Danaus plexippus L (Lepidoptera: Nymphalidae). Heredity 52, 1-7

Jelnes JE (1975a) A comparative electrophoretic study on Danish species of Aricia (Lepidoptera, Rhopalocera). Hereditas 79, 61-66

Jelnes JE (1975b) Electrophoretic studies on two sibling species Thera variata and Thera obeliscata (Lepidoptera, Geometridae) with special reference to phosphoglucomutase and phosphoglucose isomerase. Hereditas 79, 67-72

Langeron M (1949) Précis de Microscopie. Masson, Paris, $1430 p$

McNeil JN (1987) The true armyworm, Pseudaletia unipuncta: a victim of the pied piper or a seasonal migrant? Ins Sci Applic 8, 591-597
Metcalf CL, Flint WP, Metcalf RL (1962) Destructive and useful insects: their habitats and control, MCGraw-Hill, New York, 4th ed, $1087 \mathrm{p}$

Mukerii MK, Guppy JC (1970) A quantitative study of food consumption and growth in Pseudaletia unipuncta (Lepidoptera: Noctuidae). Can Ent 102, 1179-1188

Pashley DP (1983) Biosystematic study in Tortricidae (Lepidoptera), with a note on evolutionary rates of allozymes. Ann Entomol Soc Am 76, 139-148

Pierce F (1909) The genitalia of the group Noctuidae of the Lepidoptera of the British Islands. AW Ducan, Liverpool, $88 \mathrm{p}$

Pintureau B (1980a) Étude biométrique de Lymantria dispar (Lep Lymantriidae). I. Variabilité chez 3 formes. Ann Soc Ent Fr (NS) 16, 91-107

Pintureau B (1980b) Étude biométrique de Lymantria dispar (Lep Lymantriidae). II. Analyse multidimensionnelle de trois formes, spéciation et évolution. Ann Soc Ent Fr (NS) 16, 249-264

Pintureau B (1987) Systématique évolutive du genre Trichogramma Westwood (Hym Trichogrammatidae) en Europe. Thèse doctorat d'Etat, Université Paris VII, $311 p$

Pintureau B, Babault M (1981) Caractérisation enzymatique de Trichogramma evanescens et de T maidis (Hym: Trichogrammatidae); étude des hybrides. Entomophaga 26, 11-22

Pintureau B, Grenier AM, Nardon P (1991) Systematic interest and polymorphism of esterases in 3 species of Sitophilus (Coleoptera, Curculionidae). $J$ Stored Prod Res 27, 141-1249

Poitout S, Bues R (1970) Élevage de plusieurs espèces de Lépidoptères Noctuidae sur milieu artificiel riche et sur milieu artificiel simplifié. Ann Zool Ecol Anim 2, 79-91

Poitout S, Bues R (1974) Élevage de chenilles de ving-huit espèces de Lépidoptères Noctuidae et de deux espèces d'Arctiidae sur milieu artificiel simple. Particularités de l'élevage selon les espèces. Ann Zool Ecol Anim 6, 431-441

Saul SH, Sinsko MJ, Grimstad PR, Jr Craig GB (1978) Population genetics of the mosquito Aedes triseriatus: genetic-ecological correlation at an esterase locus. Am Nat 112, 333-339

Tavares J (1989) Mythimna unipuncta (Haworth) (Lep Noctuidae) aux Açores. Bioécologie et lutte biologique. Thèse doctorat d'Etat, Univ Aix-Marseille, $205 p$

Zalucki MP, Hughes JM, Carter PA (1987) Genetic variation in Danaus plexippus L: Habitat selection or differences in activity times? Heredity 59, 213-221 